\title{
How Often do Simple Dynamical Processes Have Infinitely Many Coexisting Sinks?
}

\author{
Laura Tedeschini-Lalli * and James A. Yorke \\ Department of Mathematics and Institute for Physical Science and Technology, \\ The University of Maryland, College Park, MD 20742, USA
}

\begin{abstract}
This work concerns the nature of chaotic dynamical processes. Sheldon Newhouse wrote on dynamical processes (depending on a parameter 4) $x_{n+1}=T\left(x_{n} ; \mu\right)$, where $x$ is in the plane, such as might arise when studying Poincaré return maps for autonomous differential equations in $\mathbb{R}^{3}$. He proved that if the system is chaotic there will very often be existing parameter values for which there are infinitely many periodic attractors coexisting in a bounded region of the plane, and that such parameter values $\mu$ would be dense in some interval. The fact that infinitely many coexisting sinks can occur brings into question the very nature of the foundations of chaotic dynamical processes. We prove, for an apparently typical situation, that Newhouse's construction yields only a set of parameter values $\mu$ of measure zero.
\end{abstract}

\section{Posing the Problem}

Sheldon Newhouse made some amazing discoveries about dissipative dynamical processes. His discoveries have raised questions about the nature of systems that behave chaotically. The objective of this paper is to make computations that interpret his results, and to help re-establish the foundation of chaotic dynamical processes.

We are accustomed to thinking of dynamical systems with one or several basins of attraction. For our purposes a periodic point $p$ of period $k$ for a map $T$ is called attracting if all the eigenvalues of $D\left(T^{k}(p)\right)$ are inside the unit circle in the complex plane, where the operator $D$ is the matrix of partial derivatives and $T^{k}$ is the $k^{\text {th }}$ iterate of $T$. Newhouse has shown it is possible for $T$ to have infinitely many

\footnotetext{
This research was supported in part by grants from the Air Force Office of Scientific Research AFOSR 81-0217, the Consiglio Nazionale delle Ricerche-Comitato per le Matematiche, and the National Science Foundation DMS 84-19110

* On leave from: Dipartimento di Matematica "G. Castel nuovo" Universita di Roma "La Sapienza" P. le Aldo Moro 5, I-00185 Rome, Italy
} 
attracting periodic orbits coexisting in a bounded set (Newhouse, 1974). His results suggest this may be a very common phenomenon (Newhouse, 1979). A detailed discussion of this topic along with a large related literature survey can be found in (Guckenheimer, Holmes, 1983).

In this paper, we discuss the theory of two-dimensional maps. Such maps form the basis for the understanding of low dimensional dynamical processes. For example the forced pendulum

$$
\theta^{\prime \prime}(t)+a \theta^{\prime}(t)+b \sin (\theta(t))=\varepsilon \cos (2 \pi t)
$$

can be studied using the time-one map which takes $\left(\theta, \theta^{\prime}\right)$ at time $n$ to the corresponding point at time $n+1$.

Throughout this paper we will let

$$
T(\cdot, \cdot ; \mu): \mathbb{R}^{2} \rightarrow \mathbb{R}^{2}, \quad \mu \in \mathbb{R},
$$

be a one-parameter family of area-contracting maps, where $T$ is a $C^{3}$ function, that is, $T$ is three times continuously differentiable.

Definitions. We will call values of $\mu$ for which $T$ admits infinitely many periodic attractors Newhouse values.

The attracting orbits shown to exist in Newhouse's theory are all of a special type that we call "simple." Initially (Sect. 2) orbits are called simple if for $n-1$ consecutive iterates they are near a hyperbolic fixed point. So the map is nearly linear for $n-1$ iterates and the orbit is in the region where the nonlinearity is important for the remaining 1 iterate (or perhaps a block of $k$ iterates, with $k$ fixed, $k \ll n)$. In Sect. 5 the concept is generalized replacing the fixed point with a more general hyperbolic invariant set. Correspondingly we will call $\mu$ a simple Newhouse value when $T(\cdot, \cdot ; \mu)$ admits infinitely many simple attracting orbits. These values are shown to exist in (Robinson, 1983). We argue that the set of simple Newhouse values has measure zero. At least this is true in the case presented in Sect. 5. This case appears typical. A more general derivation of this measure zero result would be desirable.

Let $p \in \mathbb{R}^{2}$ be an attracting periodic orbit of $T\left(\cdot, \cdot ; \mu_{1}\right)$. We say $p_{\mu} \in \mathbb{R}^{2}$ is the continuation of $p$, if $p_{\mu}$ is an attracting periodic orbit of $T(\cdot, \cdot ; \mu), p_{\mu}$ depends continuously on $\mu$, and $p_{\mu_{1}}=p$. Note that according to our definition, necessarily $p_{\mu}$ has the same (minimum) period as $p$. We will define the stability range $J(p)$ to be the largest interval (in $\mu$ ) on which the continuation $p_{\mu}$ of $p$ is defined and is an attractor. If $p$ and $p^{*}$ are attracting orbits for $\mu_{1}$ and $\mu_{2}$, respectively, and if they are on the same path $p_{\mu}$ of attractors, then we refer to them as being the same periodic attractor.

We assume the origin $\underline{Q}$ is a saddle fixed point for $T, T(\underline{O} ; \mu) \equiv \underline{Q}$, and we denote the smooth invariant one-dimensional manifolds of $\underline{O}$ as follows:

$$
\begin{gathered}
S_{\mu}=\left\{x \in \mathbb{R}^{2}: T^{n}(x ; \mu) \rightarrow \underline{O} \text { as } n \rightarrow \infty\right\}, \\
U_{\mu}=\left\{x \in \mathbb{R}^{2}: T^{-n}(x ; \mu) \rightarrow \underline{O} \text { as } n \rightarrow \infty\right\},
\end{gathered}
$$

$S_{\mu}$ and $U_{\mu}$ can be tangent of each other at some point, for some value of $\mu$. We will assume (see Fig. 1.1) 


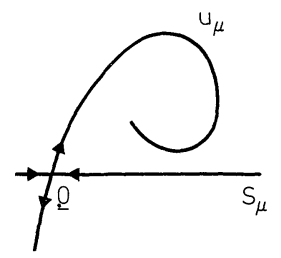

$\mu<\mu_{0}$

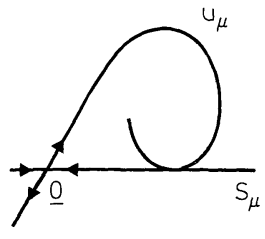

$\mu=\mu_{0}$

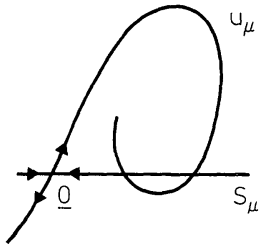

$\mu>\mu_{0}$

Fig. 1.1. As $\mu$ varies the stable and unstable manifolds of the saddle $\underline{O}$ move toward each other creating a tangency at $\mu=\mu_{0}$

i) $S_{\mu_{0}}$ is tangent to $U_{\mu_{0}}$ at $p_{0}$;

ii) the tangency is quadratic;

iii) the tangency is reached smoothly as $\mu$ varies, as detailed later. (Loosely speaking, the rate of approach of the two manifolds is bounded away from zero.) Then we say that $S_{\mu}$ and $U_{\mu}$ have a tangency at $\left(p_{0} ; \mu_{0}\right)$ that is nondegenerate in both $p_{0}$ and $\mu_{0}$, and will call $\mu_{0}$ a nondegenerate tangency value. Throughout the

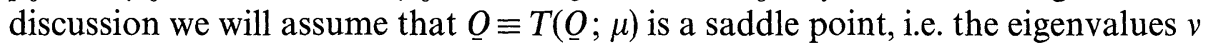
and $\lambda$ of $D T(O ; \mu)$ are real and $|v|<1<|\lambda|$ and $|v|>0$. Recall the map was assumed to be area contracting, so $|v \lambda|<1$. The following theorem was independently discovered by Newhouse (1974) and Gavrilov and Shilnikov (1972).

Theorem 1.1. Assume that $S_{\mu}$ and $U_{\mu}$ have a nondegenerate tangency at $\left(p_{0} ; \mu_{0}\right)$. Then, for all $n$ sufficiently large there is an attracting periodic point $p_{n}$ of minimum period $n$ at some $\mu_{n}$ such that $p_{n} \rightarrow p_{0}$ and $\mu_{n} \rightarrow \mu_{0}$ as $n \rightarrow \infty$, i.e. there is a family of periodic attractors approaching the tangency $\left(p_{0}, \mu_{0}\right)$.

Newhouse (1974) linked this result to the existence of diffeomorphisms with infinitely many coexisting periodic attractors, and to the abundance of such diffeomorphisms (Newhouse, 1979) by means of the following important and delicate result. We state a version from Robinson (1983).

Lemma 1.2. Let $\mu_{0}$ be a nondegenerate tangency value for $T$. Then for every $\varepsilon>0$ there is a nontrivial interval $I \subset\left(\mu_{0}-\varepsilon, \mu_{0}+\varepsilon\right)$ such that I contains a dense set of nondegenerate tangency values.

Once it is granted, as with this lemma, that nondegenerate tangency values are dense in an interval, Theorem 1.1 applies to each of them, and the next result follows.

Theorem 1.3. I contains a residual set of Newhouse values.

A residual set is the countable intersection of open dense sets; it is therefore, in particular, dense in $I$.

The above result is essentially due to Newhouse, but we state it here in Robinson's setting (Robinson, 1983) since Newhouse speaks in terms of open sets of diffeomorphisms, while Robinson speaks about one-parameter families of diffeomorphisms. The proof of Lemma 1.2 uses a construction which produces a residual set of simple Newhouse values, that is, parameter values at which there are 
infinitely many coexisting simple periodic attractors. We believe that this method of construction can produce only a set of parameters of measure zero, and we show this is true in a special case that appears quite representative.

Lemma 1.2 above is obtained by proving that there is a "hyperbolic basic set," which we can take to be the invariant set $H$ of a horseshoe map, for which every saddle $s \in H$ has a nondegenerate tangency for some $\mu=\mu(s)$. We will provide a picture of the phenomenon in Sect. 5 (Fig. 5.2). Each saddle's tangency is associated with a horde of simple orbits.

We will write $|J(p)|$ for the length of the stability range of each simple Newhouse orbit $p$. To show that the set of simple Newhouse values $N$ has measure zero, it is sufficient to prove:

$$
\sum|J(p)|<\infty,
$$

where the sum is taken over all the simple attracting periodic orbits that arise from the tangencies of all the saddles in the horseshoe. In fact, let $p_{1}, p_{2}, \ldots, p_{k}, \ldots$ be any ordering of the simple periodic sinks appearing in (1.1). The set $\mathbf{N}$ of simple Newhouse values, by definition is contained in

$$
\bigcup_{k=n}^{\infty} J\left(p_{k}\right)
$$

for every $n$. In particular, for every $n$, measure $(\mathbf{N}) \leqq \sum_{k=n}^{\infty}\left|J\left(p_{k}\right)\right|$, which can be made as small as desired if (1.1) is true, by making $n$ large. Therefore the measure of $\mathbf{N}$ is zero. (This argument is also known as Borel-Cantelli lemma.) Our main result is that (1.1) holds in the typical cases. We show it in Sect. 5 by proving that asymptotically, as $n \rightarrow \infty$, the duration $|J(p)|$ is of length proportional to $\lambda^{-2 n}$, where $n$ is the period and $\lambda$ is the expanding eigenvalue of $\underline{O}$. A further implication of this asymptotic relation is that if a simple attracting orbit exists at a randomly chosen parameter value, it is most likely to have a rather short period, since the long periods taken together have every little measure.

We emphasize that each saddle $p$ in the horseshoe $H$ gives rise to an infinity of simple attractors, and there are infinitely many saddles in a horseshoe; consideration of these attractors alone is sufficient to prove Newhouse's Theorem 1.3.

In order to obtain estimates of the stability ranges, in Sect. 2 we give an alternative proof of Theorem 1.1, which enables us to estimate the stability ranges of the family of simple periodic attractors created with this mechanism.

We found that a sequence of rescaled maps converging smoothly to the quadratic map of the interval describes the dynamics in a neighborhood of the simple attractors. The rescaling factors are linked to the eigenvalue of the saddle $\underline{O}$ and to the geometry of its manifolds at the tangency. In Sect. 3 we discuss this approach, which allows one to estimate a priori, by means of rescaling, the qualitative and quantitative behavior of each simple attractor. What information can be inferred from that of the limiting behavior is assessed in the next section.

Section 4 is devoted to a study of planar maps that are close to the scalar quadratic map, and of the conditions under which these maps have an attractor.

In Sect. 5 we describe a horseshoe that appears to be typical of the situations described by Newhouse, and we prove our conjecture that the simple Newhouse set has measure 0 in this case. 
The rigorous results of Sects. 2-4 are visualized in numerical experiments on the Hénon model, which seem to go beyond the regularity foreseen in those sections. The results of these experiments are summarized in the Appendix.

\section{An Implicit Function Theorem Proof of Theorem 1.1}

If $p$ is an attracting periodic orbit of period $m$, then for every $\mu$ in the stability range $J(p)$, each eigenvalue $\theta$ of the Jacobian $D T^{m}(\cdot, \cdot ; \mu)$ satisfies $|\theta|<1$. When $\mu$ is a boundary point of $J(p)$, the orbit $p$ still exists but has an eigenvalue $\theta= \pm 1$ that is,

$$
T^{m}\left(p_{\mu} ; \mu\right)=p_{\mu}, \quad \operatorname{det}\left(D T^{m}\left(p_{\mu} ; \mu\right)-\theta \cdot \mathrm{Id}\right)=0,
$$

where Id is the identity matrix, and $\theta= \pm 1$. Note that since we assume $T$ to be area-contracting for each $\mu, \theta= \pm 1$ are the only possibilities for an eigenvalue of $D T^{m}$ to be norm one. We are interested in periodic attractors which are intimately connected with the creation of a "tangency" of the stable and unstable manifolds.

Let $T(\cdot ; \mu): \mathbb{R}^{2} \rightarrow \mathbb{R}^{2}$ be our $C^{3}$ area-contracting map (triply differentiable also with respect to the parameter $\mu$ ), and let $\underline{O}=T(\underline{O} ; \mu)$ be a saddle point, with eigenvalues $0<v<1<\lambda$, and $|v \lambda|<1$. We assume the eigenvalues are positive only to simplify the notation. Then we can choose coordinates so that the map is linear in a neighborhood of $\underline{O}$, and assume the change of coordinates is $C^{3}{ }^{1}$ More specifically, we choose coordinates so that $T(x, y ; \mu)=(v x, \lambda y)$ when $|x| \leqq 1,|y| \leqq 1$.

We will assume that for some $\mu_{0}$ and some iterate of $T$, say $k, T^{k}\left(\cdot, \cdot ; \mu_{0}\right)$ maps some point $\left(0, Y_{0}\right)$ of the unstable manifold of $\underline{O}$, to some point $\left(x_{0}, 0\right)$ of its stable manifold, $\left(0<x_{0}<1,0<Y_{0}<1\right)$. Then $\left(0, Y_{0}\right)$ and $\left(x_{0}, 0\right)$ are called homoclinic points (see Fig. 2.1). We will write

$$
T^{k}(X, Y ; \mu)=(F(X, Y ; \mu), G(X, Y ; \mu)) .
$$

The map $(F, G)$ is also $C^{3}$ in $X, Y$ and $\mu$, and will be studied for $(X, Y ; \mu)$ in a neighborhood of $\left(0, Y_{0} ; \mu_{0}\right)$. Points written $(x, y)$ will be near $\left(x_{0}, 0\right)$ while points written $(X, Y)$ will be near $\left(0, Y_{0}\right)$ and in particular we write $(X, Y)=\left(v^{n} x, \lambda^{n} y\right)$, when $X>0, y>0$.

We will assume $T$ has a tangency at $\left(0, Y_{0}\right)$ for $\mu=\mu_{0}$, that is

i) $F\left(0, Y_{0} ; \mu_{0}\right)=x_{0}$ and $G\left(0, Y_{0} ; \mu_{0}\right)=0$ (intersection of $U_{\mu_{0}}$ and $\left.S_{\mu_{0}}\right)$;

ii) $G_{Y}\left(0, Y_{0} ; \mu_{0}\right)=0$ (tangency of $U_{\mu_{0}}$ and $\left.S_{\mu_{0}}\right)$.

We also assume the tangency is nondegenerate, i.e. $G$ also satisfies:

iii) $G_{Y Y}\left(0, Y_{0} ; \mu_{0}\right) \neq 0$;

iv) $G_{\mu}\left(0, Y_{0} ; \mu_{0}\right) \neq 0$.

Of course, $G_{Y}, G_{Y Y}, G_{\mu}$ are the partial derivatives of first and second order.

We can now phrase Theorem 1.1 in terms of bifurcations. We investigate the existence of attracting periodic points $(x, y)$ of period $n+k$ for large $n$, where the first $n$ iterates of $(x, y)$ are in the unit square and the next block of $k$ are described

\footnotetext{
${ }^{1}$ Such changes of coordinate may not exist when $\frac{\lambda}{v}$ is an integer, so we are implicitly assuming that this is not the case. See, for instance (Belitskii, 1978) or (Guckenheimer and Holmes, 1983, Chap. 5)
} 


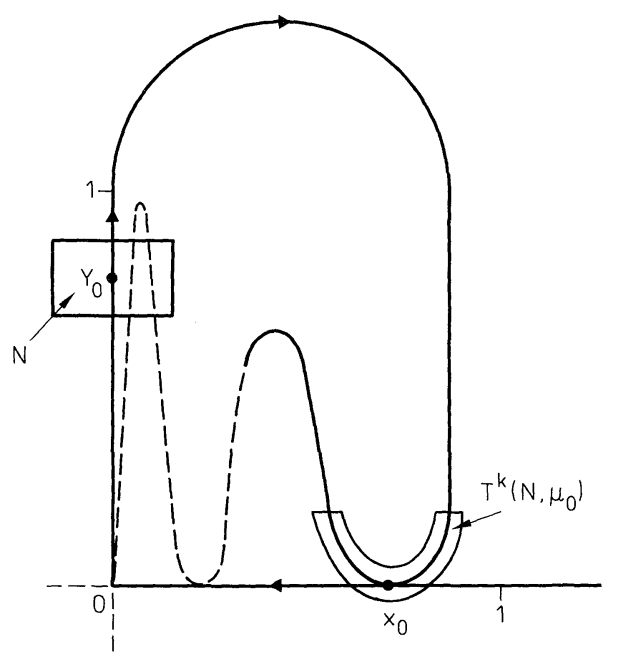

Fig. 2.1. The points $\left(x_{0}, 0\right)$ and $\left(0, Y_{0}\right)$ are points of tangency of the stable and unstable manifolds of 0 when $\mu=\mu_{0}$. For some $k>0, T^{k}\left(0, Y_{0} ; \mu_{0}\right)=\left(x_{0}, 0\right)$

more generally using $(F, G)$, so that

$$
T^{n}(x, y ; \mu)=\left(v^{n} x, \lambda^{n} y\right), \quad T^{n+k}(x, y ; \mu)=\left(F\left(v^{n} x, \lambda^{n} y ; \mu\right), G\left(v^{n} x, \lambda^{n} y ; \mu\right)\right) .
$$

Definition. We will call periodic points of this kind simple periodic orbits. Rewriting Eq. (2.1) with $m=n+k$ gives

$$
\left(v^{n} F_{X}-\theta\right)\left(\lambda^{n} G_{Y}-\theta\right)-v^{n} \lambda^{n} F_{Y} G_{X}=0 \text {. }
$$

To study the behavior near $x_{0}$, we will investigate $x+x_{0}$ for small $x$. Let $\alpha=v^{n}$, $\beta=\lambda^{-n}, X=\alpha x_{0}+\alpha x$, and $y=\beta Y$. Then,

$$
T^{n+k}\left(x_{0}+x, \beta Y ; \mu\right)=\left(F\left(\alpha x_{0}+\alpha x, Y ; \mu\right), G\left(\alpha x_{0}+\alpha x, Y ; \mu\right)\right) \text {. }
$$

Attracting fixed points of this map for $\alpha$ and $\beta$ sufficiently small, are simple attracting periodic points for $T$, of period $n+k$ where $n$ is large. Such points are zeroes of the system

$$
\Phi=\left(\Phi_{1}, \Phi_{2}, \Phi_{3}\right)=0
$$

where

$$
\begin{gathered}
\Phi_{1}(x, Y, \mu ; \alpha, \beta) \equiv F\left(\alpha x_{0}+\alpha x, Y ; \mu\right)-\left(x_{0}+x\right), \\
\Phi_{2}(x, Y, \mu ; \alpha, \beta) \equiv G\left(\alpha x_{0}+\alpha x, Y ; \mu\right)-\beta Y, \\
\Phi_{3} \equiv\left(\alpha F_{X}\left(\alpha x_{0}+\alpha x, Y ; \mu\right)-\theta\right)\left(G_{Y}\left(\alpha x_{0}+\alpha x, Y ; \mu\right)-\theta \beta\right)-\alpha F_{Y} G_{X} .
\end{gathered}
$$

The conditions $\Phi_{1}=\Phi_{2}=0$ correspond to the requirement that there is a fixed point. The condition $\Phi_{3}=0$ is equivalent to condition (2.2) when $\beta>0$. While the case $\beta=0$ has no meaning for (2.2) since $\beta=\lambda^{-n}>0$ for each $n$, it is useful to permit $\beta$ to be 0 in the above equation. While we are interested in $\theta=-1$ and $\theta=+1$, the existence of solutions will follow for all $\theta \neq 0$. 
Let $\theta$ be non-zero. The existence of solutions of $\Phi=0$, denoted

$$
x_{\theta}(\alpha, \beta), Y_{\theta}(\alpha, \beta), \mu_{\theta}(\alpha, \beta)
$$

is guaranteed by the Implicit Function Theorem which is applied to $\Phi=0$ at the trivial solution $\left(0, Y_{0}, \mu_{0}\right)$ for parameters $\alpha=\beta=0$. To see this let $P_{0}=\left(0, Y_{0}, \mu_{0} ; 0,0\right)$. The matrix of partial derivatives of $\Phi$ with respect to $(x, Y, \mu)$ has full rank at that point. In particular at $P_{0}$ (where $\left.\alpha=0\right)$

$$
\begin{gathered}
\frac{\partial \Phi_{1}}{\partial x}=\alpha F_{X}\left(\alpha x_{0}+\alpha x, Y ; \mu\right)-1=-1, \quad \frac{\partial \Phi_{2}}{\partial x}=\alpha \cdot G_{X}=0, \\
\frac{\partial \Phi_{3}}{\partial x}=\alpha^{2} F_{X X} \cdot\left(G_{Y}-\theta \beta\right)-\alpha G_{Y X}\left(\alpha F_{X}-\theta\right)-\alpha^{2}\left(F_{Y X} G_{X}+F_{Y} G_{X X}\right)=0 .
\end{gathered}
$$

Therefore the Jacobian of $\Phi$ with respect to $(x, Y, \mu)$ at $P_{0}$ is

$$
D \Phi=\left(\begin{array}{rcc}
-1 & F_{Y} & F_{\mu} \\
0 & G_{Y} & G_{\mu} \\
0 & -\theta G_{Y Y} & -\theta G_{Y \mu}
\end{array}\right),
$$

where the partials are evaluated at $\left(0, Y_{0} ; \mu_{0}\right)$. The determinant

$$
-\theta\left(G_{\mu} \cdot G_{Y Y}-G_{Y} \cdot G_{Y \mu}\right)
$$

is non-zero if $\theta \neq 0$, by the assumption of a nondegenerate tangency at $P_{0}: G_{Y}=0$, $G_{\mu} \neq 0$, and $G_{Y Y} \neq 0$. Notice the solution is on a periodic orbit only when $\alpha=v^{n}$ and $\beta=\lambda^{-n}$ are non-zero and sufficiently small, that is, for $n$ large enough. It is also clear that as $n \rightarrow \infty$, i.e. as $\alpha \rightarrow 0, \beta \rightarrow 0$, the periodic solutions and their critical values (that is $\mu$ for which $\theta= \pm 1$, and more generally any value of $\mu$ for which they are attractors), approach the trivial solution $\left(0, Y_{0}, \mu_{0}\right)$ which is by assumption one of the tangency points. Since there is a tangency at $\left(x_{0}, 0, \mu_{0}\right)$, there is also one at $\left(0, Y_{0}, \mu_{0}\right)$. Theorem 1.1 is thus proved.

\section{Estimate of the Length of the Stability Range}

For $\alpha$ and $\beta$ small the function $\mu_{\theta}(\alpha, \beta)$ is a component of a solution of (2.3) so it is $C^{3}$. The Implicit Function Theorem approach yields an estimate ${ }^{2}$ for the function

$$
\text { Length }(\alpha, \beta)=\left|\mu_{\theta=+1}(\alpha, \beta)-\mu_{\theta=-1}(\alpha, \beta)\right| \text {. }
$$

The critical estimate we need for the stability range is of the form

$$
\left|J_{n}\right|<C \lambda^{-2 n}
$$

for all $n$ sufficiently large for some constant $C$. More precisely we will prove the following proposition.

\footnotetext{
${ }^{2}$ In Sect. 4 we will see that, as one expects, a saddle-node bifurcation occurs at $\mu_{\theta}$ with $\theta=1$ and a period-doubling occurs at $\mu_{\theta}$ with $\theta=-1$
} 
Proposition 2.1. There is some $\beta^{*}>0$ and a constant $C$ for which

$$
\text { Length }(\alpha, \beta)<C \cdot \beta^{2} \text { for }|\alpha|<|\beta|<\beta^{*} \text {. }
$$

Proof. We claim

$$
\frac{\partial \mu_{\theta}}{\partial \alpha}, \frac{\partial \mu_{\theta}}{\partial \beta} \quad \text { at } \quad(\alpha, \beta)=(0,0)
$$

are independent of $\theta$. Notice Length $=0$ at $(0,0)$ since then $\mu_{\theta}$ is $\mu_{0}$ (independently of $\theta$ ). Therefore in computing Length we will be left with second order terms in $(\alpha, \beta)$, that is

$$
\text { Length }(\alpha, \beta) \leqq \text { Const } \cdot\left(\alpha^{2}+\alpha \beta+\beta^{2}\right) \text {. }
$$

Observing that $\alpha<\beta$ for our case (i.e. an area contracting map), this will then complete the proof.

To compute the derivatives of $\mu_{\theta}(\alpha, \beta)$ and show they are independent of $\theta$, consider the second equation $\Phi_{2}=0$ of problem (2.1) along a solution $\left(x_{\theta}, Y_{\theta}, \mu_{\theta}\right)(\alpha, \beta)$ :

$$
\Phi_{2}\left(x_{\theta}, Y_{\theta}, \mu_{\theta} ; \alpha, \beta\right)=G\left(\alpha x_{\theta}, Y_{\theta} ; \mu_{\theta}\right)-\beta Y_{\theta} \equiv 0 .
$$

Differentiating with respect to $\alpha$ yields

$$
0 \equiv \frac{\partial \Phi_{2}}{\partial \alpha}=G_{X} \cdot\left[x_{0}+x_{\theta}+\alpha \frac{\partial x_{\theta}}{\partial \alpha}\right]+\left[G_{Y}-\beta\right] \cdot \frac{\partial Y_{\theta}}{\partial \alpha}+G_{\mu} \cdot \frac{\partial \mu_{\theta}}{\partial \alpha} .
$$

We want to solve for $\frac{\partial \mu_{\theta}}{\partial \alpha}$ at $(\alpha, \beta)=(0,0)$ and this is possible because $G_{\mu} \neq 0$. Since $G_{Y}=0$ and $x_{\theta}=0$ at $P_{0}$, it follows that

$$
\frac{\partial \mu_{\theta}}{\partial \alpha}=-\frac{G_{X} \cdot x_{0}}{G_{\mu}}
$$

and therefore $\frac{\partial \mu_{\theta}}{\partial \alpha}$ is independent of $\theta$. Analogously,

$$
0 \equiv \frac{\partial \phi_{2}}{\partial \beta}=\alpha \cdot G_{X} \frac{\partial x_{\theta}}{\partial \beta}+\left[G_{Y}-\beta\right] \frac{\partial Y_{\theta}}{\partial \beta}+G_{\mu} \frac{\partial \mu_{\theta}}{\partial \beta}-Y_{\theta}
$$

Since $Y_{\theta}=Y_{0}$ at $(0,0)$ we have for each $\theta$

$$
\frac{\partial \mu_{\theta}}{\partial \beta}=\frac{Y_{0}}{G_{\mu}} \text { at }(0,0),
$$

thus demonstrating the claim.

Translating back to the original question our calculations now give

$$
\mu_{\theta}=\left[-v^{n} G_{X} \cdot x_{0}+\lambda^{-n} Y_{0}\right] / G_{\mu}+\text { higher order terms, }
$$

where the derivatives $G_{X}$ and $G_{\mu}$ are evaluated at $\left(0, Y_{0} ; \mu_{0}\right)$. The higher order terms depend on $\lambda^{2 n}, v^{2 n}, \lambda^{n} v^{n}$, and still higher products and might depend upon $\theta$. 


\section{The Evolution of the Period $n$ Attractor as $\mu_{0}$}

In the preceding analysis we raised the question of how long it takes for the attractor of the period $(n+k)$ saddle-node pair to lose stability as $\mu$ approaches $\mu_{0}$. Of course, there is still an attractor for some further range in $\mu$, only now the attractor has period $2(n+k)$. As $\mu$ continues, a period-doubling cascade can be expected followed by a range of generally chaotic behavior, where the chaotic orbits (Yorke and Alligood, 1983, 1985) never wander far from where the saddle-node was. The "true" interval of stability should perhaps include this entire range; we will call the length of this longer interval the "duration" of the attractor. In fact, it only increases Length by a factor of $9 / 4$ as we shall show by rescaling. ${ }^{3}$

The idea of rescaling is implicit in the standard analysis (Newhouse, 1979; Robinson, 1983), and here we investigate that idea more closely. We rescale the process using the change of variables

$$
\left.\begin{array}{l}
m=b_{n} c_{n}\left(\mu_{n}-\mu\right) \lambda^{2 n} \\
u=\left(x-x_{n}\right) \lambda^{n} \\
v=c_{n}\left(y-y_{n}\right) \lambda^{2 n},
\end{array}\right\}
$$

where $b_{n}=G_{\mu}$ and $c_{n}=\frac{1}{2} G_{Y Y}$ are evaluated at the saddle-node values

$$
S_{n} \equiv\left(v^{n} x_{n}, \lambda^{n} y_{n} ; \mu_{n}\right),
$$

that is, they only depend on $n$. We showed in the previous section that as $n \rightarrow \infty$, $S_{n} \rightarrow P_{0}$, with $P_{0}$ the nondegenerate tangency; it follows that $b_{n}$ and $c_{n}$ converge to the corresponding derivatives computed at $P_{0}$, and are therefore bounded away from zero. Applying the coordinate changes to domain and range of the map $T^{n+k}$ gives the rescaled maps

$$
\begin{gathered}
f_{n}(u, v, m) \equiv \lambda^{n}\left[F\left(v^{n} x_{n}+\frac{v^{n}}{\lambda^{n}} u, \lambda^{n} y_{n}+\frac{v}{c_{n} \lambda^{n}} ; \mu_{n}-\frac{m}{b_{n} c_{n} \lambda^{2 n}}\right)-x_{n}\right], \\
g_{n}(u, v, m) \equiv c_{n} \lambda^{2 n}\left[G\left(v^{n} x_{n}+\frac{v^{n}}{\lambda^{n}} u, \lambda^{n} y_{n}+\frac{v}{c_{n} \lambda^{n}} ; \mu_{n}-\frac{m}{b_{n} c_{n} \lambda^{2 n}}\right)-y_{n}\right] .
\end{gathered}
$$

Under change of coordinates (3.1), the map $(x, y) \rightarrow T^{n+k}(x, y ; \mu)$ becomes $(u, v) \rightarrow\left(f_{n}, g_{n}\right)$, where

$$
\begin{gathered}
f_{n}(u, v, m)=a v+\varepsilon_{n}(u, v, m), \\
g_{n}(u, v, m)=v+v^{2}-m+\eta_{n}(u, v, m) .
\end{gathered}
$$

Notice that a fixed point of the $f_{n}, g_{n}$ map is a periodic orbit of period $n+k$ for the $T$ map and that $f_{n}$ and $g_{n}$ are independent of $u$ except through terms $\varepsilon_{n}$ and $\eta_{n}$; we show these terms are small in the following proposition.

\footnotetext{
${ }^{3}$ Our original objective in this study was to examine numerically a family of simple Newhouse orbits to see in what parameter range they occurred. The bifurcation figures in the Appendix resulted from this study. These figures made it clear to us that some kind of rescaling was going on, and in short this paper is the result
} 
Proposition 3.1. If the original map $T(x, y ; \mu)$ is $C^{r}$ with $r \geqq 3$, then $\varepsilon_{n}, \eta_{n} \rightarrow 0$ in $C^{r}$ as $n \rightarrow \infty$. In other words on any bounded set in the $(u, v, m)$ space $f_{n} \rightarrow a v$. Furthermore all partial derivatives of $f$ (up to order $r$ ) converge 0 on that bounded set as $n \rightarrow \infty$ (except $\frac{\partial f_{n}}{\partial v}$ which converges to $\left.a=2 F_{Y} / G_{Y Y} \neq 0\right)$. Similarly $g_{n}$ and its partials converge to $v+v^{2}-m$ and its partials.

Proof. Equation (2.1) for the saddle-node values $\left(x_{n}, y_{n}, \mu_{n}\right)$ (i.e. for $\left.\theta=+1\right)$ may be rewritten as:

$$
\begin{gathered}
F\left(S_{n}\right)-x_{n}=0, \\
G\left(S_{n}\right)-y_{n}=0, \\
\left(v^{n} F_{X}\left(S_{n}\right)-1\right)\left(\lambda^{n} G_{Y}\left(S_{n}\right)-1\right)-v^{n} \lambda^{n} F_{Y}\left(S_{n}\right) G_{X}\left(S_{n}\right)=0,
\end{gathered}
$$

so that $\left(f_{n}, g_{n}\right)$ has a saddle-node at $u=0, v=0, m=0$ for each $n$ sufficiently large, as proved in Theorem 1.1. First notice

$$
f_{n}(0,0,0)=0=g_{n}(0,0,0) \text {. }
$$

The first partials satisfy:

$$
\begin{gathered}
\frac{\partial}{\partial u} f_{n}(u, v, m)=O\left(v^{n}\right), \\
\frac{\partial}{\partial v} f_{n}(u, v, m)=\frac{2}{c_{n}} F_{Y}=\frac{F_{Y}\left(S_{n}\right)}{G_{Y Y}}+O\left(\lambda^{-n}\right)=a+O\left(\lambda^{-n}\right),
\end{gathered}
$$

where these estimates are uniform on bounded $(u, v, m)$ sets. Hence the first partial derivatives of $f_{n}(u, v, m)$ tend to constants as $n \rightarrow \infty$, therefore (3.2) is satisfied.

The situation for the partials of $g$ is more delicate.

$$
\begin{gathered}
\frac{\partial}{\partial u} g_{n}(u, v, m)=O\left(\lambda^{n} v^{n}\right) \quad \text { and } \lambda^{n} v^{n}+0 \quad \text { as } n \rightarrow \infty, \\
\frac{\partial}{\partial m} g_{n}(u, v, m)=\frac{G_{\mu}}{b_{n}}=1+O\left(\lambda^{-n}\right), \quad \frac{\partial}{\partial v} g_{n}(u, v, m)=\lambda^{n} G_{Y},
\end{gathered}
$$

where $G_{\mu}$ and $G_{Y}$ are evaluated at

$$
\left(v^{n} x_{n}+\frac{v^{n}}{\lambda^{n}} u, \lambda^{n} y_{n}+\frac{v}{c_{n} \lambda^{n}} ; \mu_{n}-\frac{m}{b_{n} c_{n} \lambda^{2 n}}\right)
$$

Therefore

$$
\lambda^{n} G_{Y}=\lambda^{n} G_{Y}\left(S_{n}\right)+v G_{Y Y}(\bar{X}, \bar{Y} ; \bar{\mu})+O\left(v^{n} \lambda^{-n}\right)
$$

for some $(\bar{X}, \bar{Y}, \bar{\mu})$ near $S_{n}$, and of course, $G_{Y Y}$ is uniformly Lipschitz near $S_{n}$ under our assumption that $G$ and $F$ are in $C^{3}$. Recalling that $v \lambda<1$ by hypothesis, and letting $n \rightarrow \infty$ in (3.6) gives:

$$
\left(v^{n} F_{X}\left(S_{n}\right)-1\right)\left(\lambda^{n} G_{Y}\left(S_{n}\right)-1\right)=v^{n} \lambda^{n} F_{Y}\left(S_{n}\right) G_{X}\left(S_{n}\right)
$$


We see that the right-hand side is going to 0 and $v^{n} F_{X}\left(S_{n}\right) \rightarrow 0$ so

$$
\lambda^{n} G_{Y}\left(S_{n}\right) \rightarrow 1 \text { as } n \rightarrow \infty .
$$

Using (3.7) we obtain

$$
\frac{\partial g_{n}}{\partial v} \rightarrow 1+2 v \quad \text { as } \quad n \rightarrow \infty
$$

uniformly in $(u, v, m), P_{0}$.

Knowing $f_{n}(0,0,0), g_{n}(0,0,0)$ and the first partial derivatives at $(u, v, m)$ yields (3.2) and (3.3).

The error terms $\left(\varepsilon_{n}\right.$ and $\left.\eta_{n}\right)$ are $C^{r}$ small. In fact, in computing each higher order partial derivative of $f$ and $g$ at $(u, v, m)$ there are multiplicative factors, powers of $v^{n} / \lambda^{n}$ that guarantee that these functions all tend to 0 , except that $\left(\partial^{2} / \partial v^{2}\right) g_{n}(u, v, m) \rightarrow 2$.

Remark. We chose the change of coordinates (3.1) so that the limit quadratic map would have the form $q(v)=v+v^{2}-m$, which is particularly easy to study in terms of the critical values of $m$. We could have done away with the constants $c_{n}, b_{n}$ in the change of variable formulas by using the change of coordinates

$$
u=\left(x-x_{n}\right) \lambda^{n}, \quad v=\left(y-y_{n}\right) \lambda^{2 n}, \quad m=\left(\mu_{n}-\mu\right) \lambda^{2 n},
$$

which is somewhat more transparent as to the role of the expanding eigenvalue $\lambda$ in the rescaling. In this case the quadratic limit map would have the form $\tilde{q}(v)$ $=v+c v^{2}-b m$ with $c=\frac{1}{2} G_{Y Y}\left(P_{0}\right), b=G_{\mu}\left(P_{0}\right)$. In this case the critical values of $m$ are proportional to those for $q(v)$.

In the Appendix this rescaling is applied to the Hénon map.

\section{Planar Maps that are Nearly One-Dimensional Quadratics}

In this section we study maps of the plane which are $C^{3}$ perturbations of the onedimensional quadratic map and the conditions under which they have an attractor. This will yield, in particular, the information we want about the evolution of simple periodic attractors that are created in the neighborhood of a nondegenerate tangency; such attractors, in fact, are described by (3.2), (3.3) via the change of coordinates (3.1).

Let a map $M$ of the $(u, v)$-plane be defined by

$$
M(u, v ; \varepsilon, m)=Q(v ; m)+P(u, v ; \varepsilon, m),
$$

where $Q(v ; m)$ is the often studied quadratic map $Q(v ; m)=\left(v, v+v^{2}-m\right)$. Note the lack of dependence on $u$; of course, the dynamics of $Q$ are exactly those of $v \rightarrow q(v ; m)$ where $q(v ; m)=v+v^{2}-m$.

In our case the perturbation $\|P(u, v ; \varepsilon, m)\|$ goes to 0 in $C^{3}$ as $\varepsilon$ goes to 0 , and in particular we assume the convergence to 0 is uniform on any bounded disk in the $(u, v)$ plane. We also assume the Jacobian of $M(u, v ; \varepsilon, m)$ is nonsingular whenever $\varepsilon \neq 0$. 


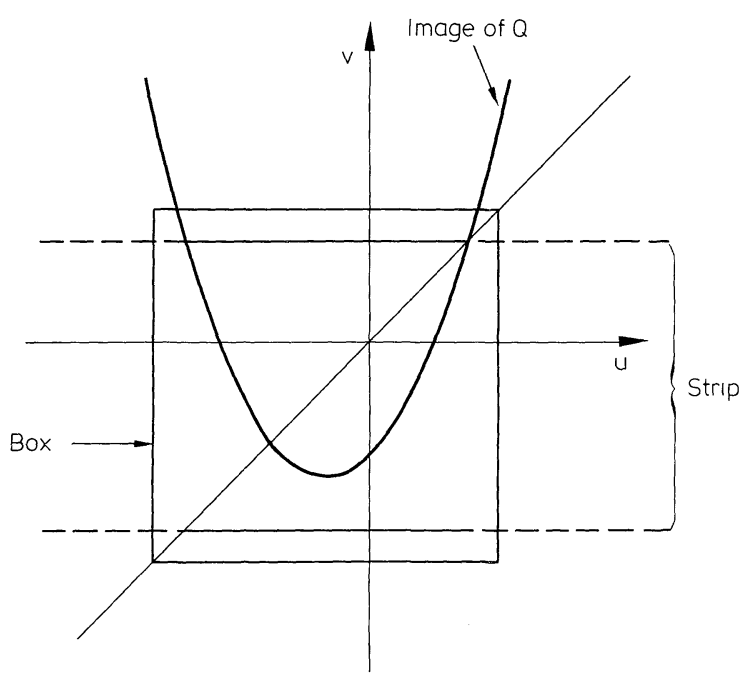

Fig. 4.1. The unperturbed quadratic map $Q(u, v)$ is $\left(v, v+v^{2}-m\right)$, pictured for $m$ in $(0,9 / 4)$. Box contains all bounded attractors of $Q$. All trajectories starting outside Strip diverge monotonically. Horizontal lines map to points on the parabola

We will prove that the range of parameters $m$ for which $M(\cdot, \cdot ; \varepsilon, m)$ has an attractor [i.e. an attractor of the process $(u, v) \rightarrow M(u, v ; \varepsilon, m)$ ], is approximately the same as for the map $Q$, when $\varepsilon$ is sufficiently small.

There are many ways to define an attractor. For our purposes we will say that a closed invariant set $A$ is an attractor for the map $M$ if the following conditions are satisfied:

i) $A$ is "Lyapunov stable," i.e. every open neighborhood $D$ of $A$ contains an open neighborhood $D^{\prime}$ of $A$ whose image under $M$ is still in $D: D \supset M\left(D^{\prime}\right) \supset A$.

ii) $A$ is "attracting," i.e. there is an open neighborhood $B$ of $A$ whose points are attracted to $A$ under iteration of $M$ :

$$
\operatorname{dist}\left(M^{n}(p), A\right) \rightarrow D \quad \text { as } \quad n \rightarrow \infty \text { for each } \quad p \in B .
$$

We restrict attention to the behavior of trajectories on any large bounded subset of $\mathbb{R}^{2}$. Consider (Fig. 4.1) the square box:

$$
\text { Box }=\left[-\frac{5}{2}, \frac{3}{2}\right] \times\left[-\frac{5}{2}, \frac{3}{2}\right] \text {. }
$$

This box contains all the bounded attractors of $Q$ as $m$ varies. Consider now an arbitrarily large closed square box called Bigbox containing Box in its interior. Then inside Bigbox the evolution of attractors of $M(u, v ; \varepsilon, m)$ is quite predictable, as the following results show.

Proposition 4.1. If $m<0$ then for \& sufficiently small every trajectory leaves Bigbox.

Proposition 4.2. If $m>9 / 4$ then for $\varepsilon$ sufficiently small almost every trajectory leaves Bigbox. 
Therefore when $m$ is not in $[0,9 / 4]$ and $\varepsilon$ is sufficiently small, Bigbox does not contain any attractors. When $m$ is in $(0,9 / 4)$ it does; more precisely:

Proposition 4.3. i) If $m \in(0,1)$, then for $\varepsilon$ sufficiently small, Box contains an attracting fixed point;

ii) if $m \in(0,9 / 4)$, then for $\varepsilon$ sufficiently small, Box contains an attractor, $A(\varepsilon)$. Furthermore for $\varepsilon$ sufficiently small, each point in Bigbox either is attracted to $A(\varepsilon)$ or eventually leaves Bigbox.

Proof. of 4.1. If $m<0$ then $q(v ; m)>v+|m|$ for all $v$; hence for $\varepsilon=0$, every point $(u, v)$ in Bigbox is the initial point of an unbounded trajectory, and the compactness of Bigbox implies every trajectory must leave Bigbox. For $\varepsilon$ small enough (depending on $m$ and on the size of Bigbox), by continuity, every trajectory must leave Bigbox.

To prove, as in Proposition 4.2, that Bigbox does not contain an attractor for certain values of $m$ and $\varepsilon$, we rely on properties stemming from the expansiveness of hyperbolic sets, and in particular of horseshoes and refer to the original articles for details.

Lemma 4.4. Let $m>9 / 4$, and $I_{m}=[-1-\sqrt{m}, \sqrt{m}]$ (see Fig. 4.2). Then there exists an integer $k=k(m)$ and a bound $\xi>1$ such that if $q^{i}(v ; m) \in I_{m}\left(i . e .\right.$, the $i^{\text {th }}$ iterate) for all $i=0,1, \ldots, k$, then $\left|\frac{d}{d v} q^{k}(v ; m)\right|>\xi$.

Lemma 4.4 implies that $q(v ; m)$ is "expanding" for $m>9 / 4$, and that almost all trajectories leave $I_{m}$ (see Nusse, 1983; Henry, 1973). In fact, for almost every initial point $v$ in $I_{m}, q^{j}(v ; m) \rightarrow \infty$ as $j \rightarrow \infty$. We may say heuristically that $q(v ; m)$ is a onedimensional horseshoe map. We must prove an analogous result for the map $M(u, v ; \varepsilon, m)$.

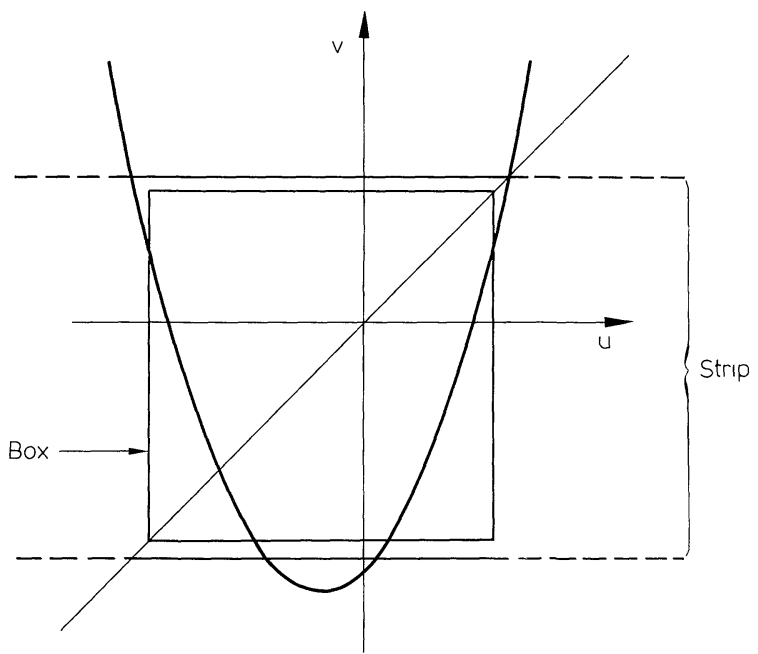

Fig. 4.2. For $m>\frac{9}{4}$ the interval $I_{m}=[-(1+\sqrt{m}), \sqrt{m}]$ is not invariant under $q$ and therefore Strip is not invariant for $Q$. Moreover, as before, anything outside Strip goes to infinity under iteration 
Lemma 4.5. Let $m>9 / 4$ and let $k=k(m)$ and $\xi>1$ be given by Lemma 4.4. Let

$$
\Omega(\varepsilon)=\left(\underline{p}: M^{n}(\underline{p} ; \varepsilon, m) \text { is in Bigbox for all } n=0, \pm 1, \pm 2, \ldots\right)
$$

then the map $M^{k}(\cdot ; \varepsilon, m)$ is uniformly hyperbolic on $\Omega(\varepsilon)$ for $\varepsilon$ sufficiently small.

Proof of Lemma 4.5. First observe that $M^{k}(\cdot, \cdot ; \varepsilon, m)=Q^{k}(\cdot, \cdot ; m)+\widetilde{P}(\cdot, \cdot ; \varepsilon, m)$, where $\|\tilde{P}(\cdot, \cdot ; \varepsilon, m)\|$ goes to 0 in $C^{3}$ as $\varepsilon$ goes to 0 . Let $A_{m}^{k}=\left\{v \in I_{m}: q^{j}(v ; m) \in I_{m}\right.$ for all $j=0, \ldots, k\}$; since $Q^{k}$ is the two-dimensional version of $q^{k}$, by Lemma 4.4 it is hyperbolic over the set $A_{m}^{k} \times A_{m}^{k}$, and in fact over the whole set $\mathbb{R} \times A_{m}^{k}$, which contains $\Omega(0)$. The image of $Q^{k}$ is a curve, $\Gamma_{k}$ and in $\Gamma_{k} \cap$ Box the expanding direction is the tangent to the curve $\Gamma_{k}$. The contracting direction for $Q^{k}$ is the horizontal line through the given point. To prove that $M^{k}(\cdot ; \varepsilon)$ is hyperbolic for $\varepsilon$ small, it suffices to construct sectors with sufficiently small size $\alpha$ (see Fig. 4.3) so that the sector "sides" have slope smaller than $\Gamma_{k}$. We find $D M^{k}$ maps the vertical sector at a point $(u, v)$ strictly into the vertical sector of $M^{k}(u, v ; \varepsilon, m)$. Furthermore, since by Lemma $4.4, Q^{k}$ is expanding vertically at those points of Box that are mapped into Box, the vertical sectors are elongated vertically by the map $D M^{k}$. The horizontal sectors have the analogous property under $D M^{-k}$. It follows from standard arguments of perturbations of sectors (Nitecki, 1971, Sects. 4.4 and 4.5) that the map is hyperbolic on the invariant set in Box.

Proof of 4.2. Lemma 4.5 guarantees that $M^{k}$ is a horseshoe map on Strip $=\mathbb{R} \times I_{m}$. Bowen and Ruelle (1975) prove that if $T:$ Box $\times \mathbb{R}^{2}$ is a $C^{2}$ horseshoe, then almost every trajectory leaves Box. More precisely and more generally, they prove that if $T$ is a $C^{2}$ Axiom- $A$ diffeomorphism, then each basic set either contains an attractor (not true in our case because we have a horseshoe map) or the measure of its stable manifold of its nonwandering set is 0 . (In our case the stable manifold of the nonwandering set is the set of points whose trajectories remain bounded for all future time.) Bowen (1975) also constructs a "fat" $C^{1}$ horseshoe showing the necessity of the $C^{2}$ condition. In our case Proposition 4.2 follows from their result, and from observing that for $\varepsilon$ small enough $M^{k}(\cdot ; \varepsilon)$ is a horseshoe over Strip, and that points in Bigbox outside Strip must leave Bigbox. Therefore almost every point in Bigbox eventually leaves it. So there are no attractors in Bigbox.

Proof of Proposition 4.3. i) For $\varepsilon=0$, the map $Q$ has an attracting fixed point in Box, for $m$ in $(0,1)$. At $m=0$ the point appears via a saddle-node bifurcation, and it loses stability at $m=1$ with a period-doubling bifurcation. A generic perioddoubling bifurcation cannot be destroyed by small $C^{3}$ perturbations. The bifurcation condition det $\left[\frac{\partial M}{\partial p}(p(\varepsilon, m)) \pm \mathrm{Id}\right]=0$ (as at beginning of Sect. 1$)$ is said
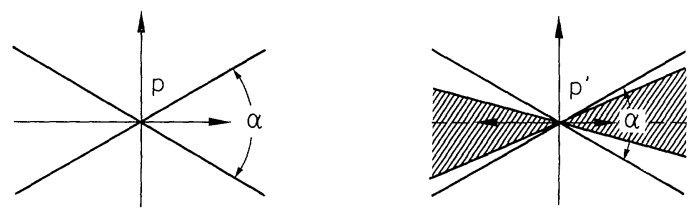

Fig. 4.3. The horizontal sector of size $\alpha$ is mapped strictly inside the horizontal sector of size $\alpha$ at $p^{\prime}$ under $D M^{k}$ 
to be generic if some conditions of transversality with respect to the parameters $m$ and $\varepsilon$ are met. Such conditions are met by the map $Q$ at $m=0$ and $m=1$. The transversality conditions with respect to $m$, in turn imply the existence of (continuous) curves $m_{s}(\varepsilon)$ and $m_{d}(\varepsilon)$ of bifurcation for $\varepsilon$ small enough, and thus the existence of an attracting fixed point for $M(\cdot, \cdot ; \varepsilon, m)$ when $m$ is between $m_{s}(\varepsilon)$ and $m_{d}(\varepsilon)$. For an analytical approach leading to the existence of bifurcation curves, see (Chow and Hale, 1982), for a more geometrical, and more compact exposition of the concepts and results about generic saddle-node and period-doubling bifurcations (see Guckenheimer and Holmes, 1983).

ii) For $\varepsilon$ small, $M(\operatorname{Box} ; \varepsilon, m)$ is in the interior of Box. It follows that $A(\varepsilon)$ $=\bigcap_{n=1}^{\infty} M^{n}(\mathrm{Box} ; \varepsilon, m)$ is a compact set that is Lyapunov stable and attracts all points in Box. In other words it is an attractor. If the second claim in 4.3ii) was false, there would exist $\varepsilon_{i} \rightarrow 0$ and points $x_{i}$ in Bigbox for which $M^{n}\left(x_{i} ; \varepsilon_{i}\right)$ remains in Bigbox and does not converge to $A\left(\varepsilon_{i}\right)$. For $\varepsilon$ small $A\left(\varepsilon_{i}\right)$ attracts all points in Strip, so $M^{n}\left(x_{i} ; \varepsilon_{i}\right)$ is never in Box. We may assume without loss of generality that $x_{1}$ has a limit $x_{o}$ in Bigbox with $x_{0}$ not in the interior of Box. Furthermore $M^{n}\left(x_{i} ; \varepsilon_{i}\right)$ $\rightarrow M^{n}\left(x_{0} ; 0\right)$, so $M^{n}\left(x_{0} ; 0\right)$ is not in the interior of Box. However there is no such initial point for $\varepsilon=0$ and $m$ in $(0,9 / 4)$; so the result is proved.

Conclusions. We can now apply this study to the sequence of maps (3.2),(3.3) which for $n$ large enough are $C^{3}$ close to $Q(v ; m)$; in this case $\varepsilon=\lambda^{-n}$. For $n$ sufficiently large a simple periodic sink is created via a saddle-node bifurcation at $m_{1}(n)=O_{1}\left(\lambda^{-n}\right)$, and it loses stability via period-doubling bifurcation at $m_{2}=1+O_{2}\left(\lambda^{-n}\right)$. The map continues to have an attractor in Box for values of $m$ up to $m_{c}(n)=\frac{9}{4}+O_{3}\left(\lambda^{-n}\right)$ when the simple attractor is destroyed.

Translating these estimates to our original map $T^{n+k}(x, Y ; \mu)$ in the neighborhood of a simple periodic orbit with the change of coordinates inverse of (3.1) we get

$$
\frac{\mu_{c}(n)-\mu_{1}(n)}{\mu_{2}(n)-\mu_{1}(n)}=\frac{9}{4} \lambda^{-2 n}+O\left(\lambda^{-3 n}\right)
$$

\section{Measure of the Newhouse Set}

So far, like Newhouse, we have proved that a nondegenerate tangency value $\left(p_{0}, \mu_{0}\right)$ of a saddle is necessarily associated with a family of sinks $\left(p_{n}, \mu_{n}\right)$ approaching the tangency, and unlike Newhouse we have estimated their stability ranges. Note that these sinks do not coexist; that is, their stability ranges do not overlap.

The argument (Newhouse, 1974; Robinson, 1983) for the existence of Newhouse values relies on having infinitely many different tangencies in an interval of the parameter $\mu$ near $\mu_{0}$, and thus on having a collection of families of periodic attractors.

In this section we extend the ideas of Sect. 2 to particular horseshoe maps (for reference on horseshoe maps see Guckenheimer and Holmes, 1983, Chap. 5). In this case the unit neighborhood in which the map is essentially linear is replaced by the rectangle $L$, as in Fig. 5.1. There are, of course, infinitely many periodic saddles 


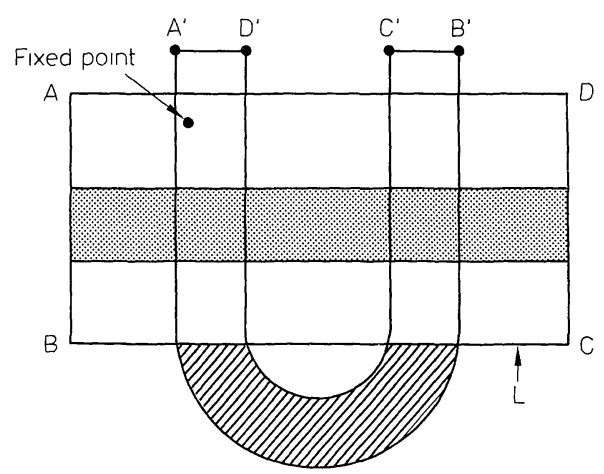

Fig. 5.1. A horseshoe map. The dotted region gets mapped to the shaded region, which is outside of $L . A^{\prime} B^{\prime} C^{\prime} D^{\prime}$ are the images of $A, B, C, D$, respectively

lying wholly within $L$. Each of these can play the role of the saddle fixed point in Sects. 2 and 3. For each period $n$ saddle in $L$ we will see there can be a periodic sink of period $n+k$ (where, as before, $k$ is fixed and $n$ is sufficiently large); the trajectory of the periodic sink is in $L$ for (at least) $n$ consecutive iterates. We will call such orbits simple orbits of the horseshoe. "Simple" refers to the fact that they lie outside $L$ only for a single block of at most $k$ iterates in each period. In Sect. 2 we showed there is a simple attractor of period $n+k$, arising from the tangency of one saddle fixed point; here there can be many simple attractors of the horseshoe, all with period $n+k$. The precise description of simple orbits in this more general case will become clearer as notation is developed.

Our objective is to estimate the durations of the simple attractors of the horseshoe, for a special map for which the computation is feasible and to show that the sum of the durations of all these simple attractors is finite. Newhouse uses only these attractors in constructing the simple Newhouse values. It will follow, as discussed in Sect. 1, that the resulting simple Newhouse values form a set of measure 0 , because it can be covered by a collection of open intervals the sum of whose lengths is arbitrarily small. The open sets will be the durations of the attractors.

Let $L$ be a rectangular region of $\mathbb{R}^{2}$ whose image under $T$ is horseshoe-shaped as in Fig. 5.1. Under some assumptions of smoothness and hyperbolicity, one can show (see Nitecki, 1971, Chap. 4; Bowen and Ruelle, 1975) that almost all points in $L$ eventually leave $L$, and that the set of points staying in $L$ for all iterates of $T$ is the product of two Cantor sets that we will denote

$$
C=\left\{p \in L: T^{n}(p ; \mu) \text { is in } L \text { for all } n=0, \pm 1, \pm 2, \ldots\right\} .
$$

The periodic points for $T(\cdot ; \mu)$ whose trajectories lie entirely in the box $L$ are dense in $C$ (Nitecki, 1971, Chap. 4), they are necessarily all saddles, and for each $n$ there are $2^{n}$ points $p$ fixed by $T^{n}$ in $L$.

Two examples of systems with a large set of tangencies arising out of a horseshoe are shown in Fig. 5.2. It is necessary to follow the extension of the unstable manifolds of the periodic points in $C$ pictured in Fig. 5.2. Newhouse finds 


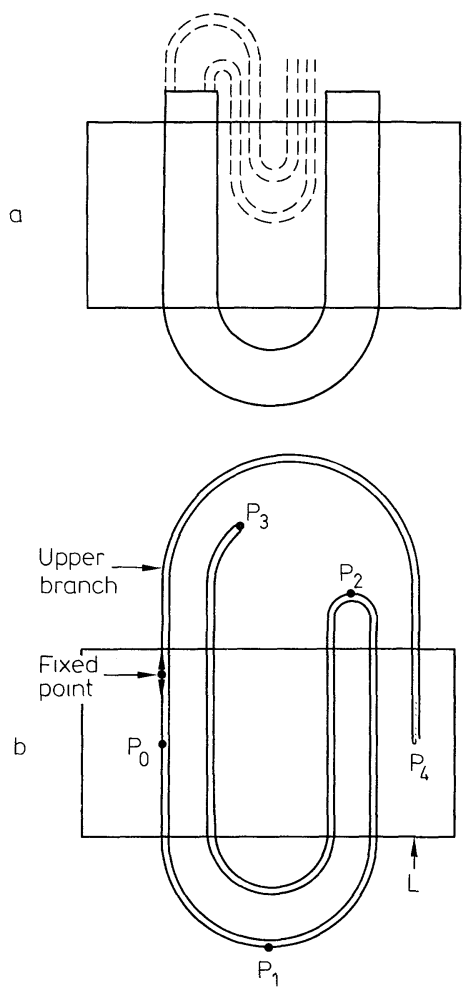

Fig. 5.2. a One way in which a horseshoe can yield numerous homoclinic tangencies. b Another way: if the upper branch of the unstable manifold of the fixed point passes through the box $L$, many tangencies may be expected. The orbit $P_{0} \rightarrow P_{1} \rightarrow \ldots \rightarrow P_{4}$ illustrates the dynamics of the map

a special horseshoe, whose periodic saddles each have a nondegenerate tangency value, and taken together these parameter values are dense in some interval (see Newhouse, 1979; Robinson, 1983 for details).

We will construct a particular horseshoe (the standard horseshoe in fact), for which the tangencies and their associated attractors can be explicitly described. We believe that our computations of durations are still true in outline for more general horseshoes. A horseshoe with features similar to these is thoroughly described in (Gambaudo and Tresser, 1983).

For each point $p$ in $L$ let $V(p)$ denote the vertical line segment in $L$ containing $p$, and $H(p)$ the horizontal segment in $L$ containing $p$.

We will assume (as in Fig. 5.3a):

i) $T(\cdot ; \mu)$ is linear (and independent of $\mu$ ) on each of the two strips in $L$ whose image is in $L$, that is on $T(L ; \mu) \cap L$. On one of these the Jacobian $D T(\cdot ; \mu)$ is $\left(\begin{array}{rr}-v & 0 \\ 0 & -\lambda\end{array}\right)$; on the other it is $\left(\begin{array}{ll}v & 0 \\ 0 & \lambda\end{array}\right)$, where $0<v<1<\lambda$. Notice that for every $p$ in $C$

$H(p)$ lies on the stable manifold of $p$,

$V(p)$ lies on the unstable manifold of $p$. 

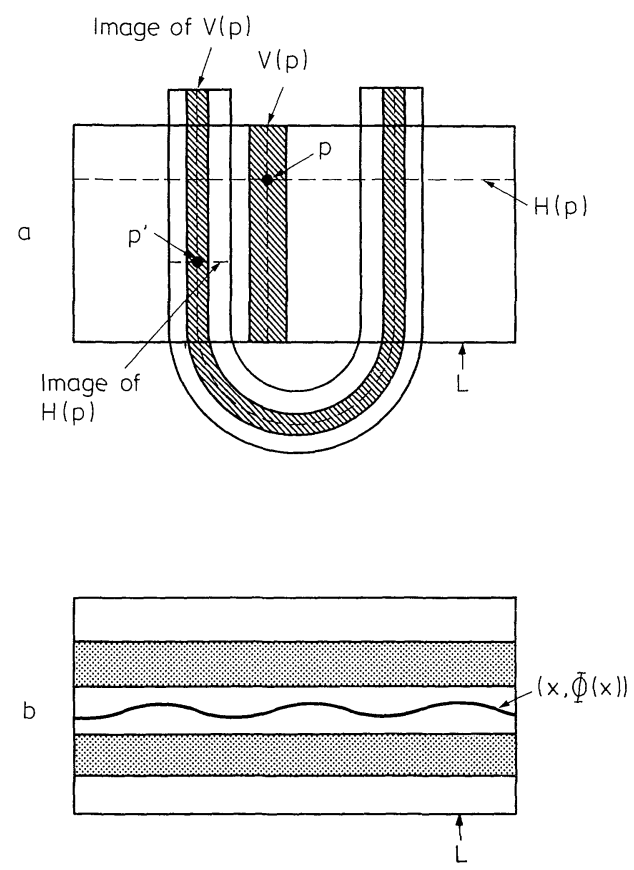

Fig. 5.3. a Our "linear" horseshoe map. The vertical segment $V(p)$ through $p$ is mapped to a curve containing the vertical segment through $p^{\prime}$, where $p \rightarrow p^{\prime}$. The horizontal segment $H(p)$ is mapped to a horizontal segment contained in $H\left(p^{\prime}\right)$. The image of a vertical strip intersected with $L$ is two vertical strips. $\mathbf{b}$ The dotted regions are the ones mapped in $L$ under one iteration of $T$. The curve $(x, \phi(x))$ is mapped outside of $L$

ii) There is a curve $(X, \phi(X))$ of points in $L$ that maps outside $L$ (and therefore does not intersect $C$ ), whose $k^{\text {th }}$ iterate is again in $L$ (as in Fig. 5.3b). In a neighborhood of the curve we assume $T^{k}(\cdot ; \mu)$ has the form

$$
T^{k}((X, Y) ; \mu)=(F(X, Y), G(X, Y)+\mu)
$$

and also

$$
\frac{\partial G}{\partial Y}(X, \phi(X))=0, \quad \frac{\partial^{2} G}{\partial Y^{2}}(X, \phi(X)) \neq 0
$$

Notice the particularly simple dependence of $T^{k}$ on $\mu$ when $(X, Y)$ is near the curve. It follows that if $(X, Y)$ is a periodic point in $C$, the point $(X, \phi(X))$ will for some $\mu$ be mapped to the stable manifold $H((X, Y))$ and, from (5.1), will have a nondegenerate tangency for that $\mu$.

For each point $p$ for which $p, T(p ; \mu), \ldots, T^{n}(p ; \mu)$ are all in $L$, we have

$$
D\left(T^{n}(p ; \mu)\right)= \pm\left(\begin{array}{cc}
v^{n} & 0 \\
0 & \lambda^{n}
\end{array}\right)
$$


and $T^{n}$ is linear on a horizontal strip stretching all the way across $L$ and containing $p$; that is, there are constants $(\bar{x}, \bar{y})$ so that on the thin horizontal strip we can write $T^{n}(\cdot ; \mu)$ as

$$
(x, y) \rightarrow\left(\alpha x+(1-\alpha) \bar{x}, \beta^{-1} y+\left(1-\beta^{-1}\right) \bar{y}\right),
$$

where $\alpha= \pm v^{n}$ and $\beta= \pm \lambda^{n}$, as in Sect. 2. Notice $(\bar{x}, \bar{y})$ is the fixed point of (5.2) and must lie in the strip.

We now compose $(F, G)$ with the linear map in (5.2), a procedure mimicking what we did in Sect. 2. Write $Y$ for $\beta^{-1} y+\left(1-\beta^{-1}\right) \bar{y}$ as in Sect. 2 the Implicit Function Theorem is applied to:

$$
\left.\begin{array}{r}
F(\alpha x+(1-\alpha) \bar{x}, Y)-x=0 \\
G(\alpha x+(1-\alpha) \bar{x}, Y)+\mu-(\beta Y+(1-\beta) \bar{y})=0 \\
\alpha\left(F_{X}-\theta\right)\left(G_{Y}-\beta \theta\right)-\alpha F_{Y} G_{X}=0
\end{array}\right\}
$$

for $|\theta| \leqq 1, \theta \neq 0$.

We want to prove (5.3) has solutions $(x, Y)$ (that depend on $\bar{x}, \bar{y}$ and $\alpha, \beta)$. Then $((x, \beta Y+(1-\beta) \bar{y})$ is a periodic point of $T$ for certain choices of $\bar{x}, \bar{y}, \alpha, \beta$. Notice that in (5.3) $\bar{x}, \bar{y}$ can be viewed as any point in $L$. When $\alpha=v^{n}, \beta=\lambda^{-n}$ and when $(\bar{x}, \bar{y})$ is a periodic saddle of period $n$, this solution corresponds to a periodic point for $T$ which is a sink for some $\mu$. When $|\theta|<1$ and $\theta \neq 0$, the solution would be an attractor, but we are especially concerned with $\theta= \pm 1$.

Problem (5.3) is satisfied at $\alpha=\beta=0$ by the point $x=\bar{x}, Y=\phi(\bar{x}), \mu=\mu_{0}(\bar{x}, \bar{y})$ $\equiv \bar{y}-G(\bar{x}, \phi(\bar{x}))$. The Jacobian of the left-hand side of (5.3) has full rank at this point, by the assumptions we made on $(x, \phi(x))$, which are analogous to the nondegeneracy of the tangency in Theorem 1.1. From the Implicit Function Theorem, for each $(\bar{x}, \bar{y})$ and $\theta \neq 0$ there exists a solution of system (5.3) for $\alpha$ and $\beta$ sufficiently small. Indeed, $\alpha>0$ and $\beta>0$ can be chosen small enough so that there will be solutions for all $(\bar{x}, \bar{y})$ in $L$. This follows from the compactness of $L$ and the smoothness of $T$. Therefore for $n$ large enough, there is such a sink. Moreover for every $n$, if $p_{m}$ in $L$ is a periodic point of period $m=n+k$, which stays inside $L$ for $n$ iterates and after $k$ iterates it has come back under the action of $(F, G+\mu)$, then it is always possible to choose $(\bar{x}, \bar{y})$ to be a periodic saddle of period $n$ whose orbit is entirely in $L$, and follows the same + and - pattern for the DT inside $L$, as holds for $D T^{i}\left(p_{m}\right)$. Therefore $p=(x, y)$ satisfies (5.3).

Propositions 4.1 and 4.2 imply that the duration (i.e. the length of the parameter range) of the attractor for $(3.2,3.3)$ is $9 / 4$, so using the scale change (3.1) the duration of our attractor is,

$$
J\left(p_{n}\right)=\frac{9}{4} \lambda^{-2 n}\left[G_{Y Y}(x, \phi(x))\right]^{-1}+O\left(\lambda^{-3 n}\right),
$$

for $n$ large enough, since in this case we have $G_{\mu} \equiv 1$; the error term $O\left(\lambda^{-3 n}\right)$ is uniformly small in the choice of $p_{n}$, due to the regularity of (5.3) and the compactness of $L$.

Remark. Newhouse restricts attention to the tangencies of the fixed point's stable and unstable manifolds. These yield only some simple attractors of period $+k$, those that stay in $L$ for $n$ iterates and approach close to the fixed point while in $L$. 
For our computational point of view, it is easier for us to sum over all the simple orbits of the horseshoe, including even those that do not approach the origin.

Theorem 5.1. Let $N$ be the set of simple Newhouse values in the interval of $\mu$ in $R$ so that $T(\cdot ; \mu)$ is described by our horseshoe on L. Then $N$ has zero Lebesgue measure.

To evaluate the measure of simple Newhouse values, as stated in Sect. 1 we can sum the durations of all the simple sinks of the horseshoe.

Proof of Theorem 5.1. As shown in Sect. 1, the result will follow from proving

$$
\sum\left|J\left(p_{n}\right)\right|<+\infty,
$$

where the sum is taken over all the simple sinks arising from all the tangencies of the horseshoe, that is, the tangencies of the horseshoe, that is, the tangencies of all the saddles in $C$. We will write $N_{n}$ for the number of saddles in $C$ of period $n$ and

$$
\left.K=\frac{9}{4} \cdot \sup _{x} \mid G_{Y Y}(x, \phi x)\right) \mid,
$$

where sup is restricted to the $x$ for which $(x, \phi(x))$ is in $L$. Then (5.5) becomes

$$
K \cdot \sum_{n=1}^{\infty} N_{n} \cdot\left(\lambda^{-2 n}+O\left(\lambda^{-3 n}\right)\right) \text {. }
$$

The number of period $n$ points in $C$ is $2^{n}$ (Nitecki, 1971), therefore since $\lambda>2$ for a linear horseshoe, the sum of durations satisfies

$$
\sum\left|J\left(p_{n}\right)\right| \leqq K \cdot \sum_{n=1}^{\infty} 2^{n}\left(\lambda^{-2 n}+O\left(\lambda^{-3 n}\right)\right)<\infty .
$$

\section{Understanding the Limitations of Our Results}

The stable orbits constructed by Newhouse all lie in $L$ for $n$ iterates and are outside for at most $k$; so the orbits he considers include only the ones we study. Both approaches ignore attractors which are not "simple," and we have not ruled out the possibility that if the durations of all the attracting periodic orbits were added, the sum of their durations would be infinite. On the other hand, the only reason for expecting Newhouse values to be common is Newhouse's proof, and we have shown here that that proof produces sets of measure zero. While we have done this only for the standard linear horseshoe, we would be amazed if simple Newhouse values have positive measure in any other contexts.

We have assumed that the map is linear on each of the two rectangles containing $C$. We then show that the simple periodic orbits of period $n+k$ have duration

$$
\frac{G_{Y Y}}{G_{\mu}} \cdot \lambda^{-2 n}+O\left(\lambda^{-3 n}\right) .
$$

The importance of the linearity assumption is not to obtain this formula. In general $G_{Y Y} / G_{\mu}$ would, of course, have to be replaced by a related value. The linearity 
allows us to conclude that the $O\left(\lambda^{-3 n}\right)$ error terms are uniformly small for each large $n$ and that the sum of the terms (6.1) over all simple orbits is finite.

Notice that in our formulas we use the fact that $\lambda>2^{1 / 2}$. If the expansion rate along the unstable manifolds varies, we would only require that these rates are uniformly greater than $2^{1 / 2}$. The Newhouse construction in fact gives all these rates greater than 2 .

\section{Appendix: Simple Newhouse Orbits in the Hénon Model}

The Hénon model

$$
T(x, y ; \mu, B)=\left(1-\mu x^{2}+y, B x\right)
$$

is well studied in the literature, usually fixing $B=0.3$ and letting $\mu$ vary. Homoclinic points (i.e. intersections of the stable and unstable manifolds of a fixed point) have been proved to exist for $\mu=1.4$. See Franceschini and Russo (1981), which also display exhaustive pictures of the two manifolds and see Misiurewicz (1980). As $\mu$ increases, tangencies are created that numerically appear to be nondegenerate. In particular $\mu \simeq 1.15$ is the first tangency value for a fixed point. At this value a two-piece chaotic attractor merges into a one-piece attractor.

This paper began with a numerical investigation of the family of simple periodic orbits associated with this tangency. Notice the determinant of the

Table 1. $\mu_{0}=1.153569796$ is the first tangency value for a fixed point. $\mu_{1}$ denotes the saddle-node value for the period $n$ orbit, while $\mu_{2}$ denotes its period-doubling parameter value, and $\mu_{c}$ is the point at which the associated chaotic attractor and its basin are destroyed. For the scalar quadratic map $\frac{\mu_{c}-\mu_{1}}{\mu_{2}-\mu_{1}}=2.25$. While the eigenvalue $\lambda$ varies with $\mu$, we use the eigenvalue $\lambda_{0}=-1.732432$ calculated at tangency value $\mu_{0}$. The products $\left(\mu_{1}-\mu_{0}\right) \cdot \lambda^{n}$ and $\left(\mu_{c}-\mu_{1}\right) \cdot \lambda^{2 n}$ are shown to converge to constants as $n \rightarrow \infty$ in Sects. 3 and 4

\begin{tabular}{|c|c|c|c|c|c|c|c|c|}
\hline \multirow{2}{*}{$\begin{array}{l}\mathrm{Pe}- \\
\text { riod } \\
n\end{array}$} & \multicolumn{4}{|c|}{ Saddle-node } & \multirow{2}{*}{$\begin{array}{l}\begin{array}{l}\text { Stability } \\
\text { range }\end{array} \\
\mu_{2}-\mu_{1}\end{array}$} & \multicolumn{3}{|l|}{ Duration } \\
\hline & $x$ & $y$ & $\mu_{1}-\mu_{0}$ & $\left(\mu_{1}-\mu_{0}\right) \lambda^{n}$ & & $\mu_{c}-\mu_{1}$ & $\frac{\mu_{c}-\mu_{1}}{\mu_{2}-\mu_{1}}$ & $\left(\mu_{c}-\mu_{1}\right) \lambda^{2 n}$ \\
\hline 6 & 0.8444863 & 0.3906411 & 0.091197949 & -2.4656 & 0.008699516 & 0.018328153 & 2.107 & 13.40 \\
\hline 8 & 0.9323534 & 0.3903470 & 0.031734043 & -2.5750 & 0.000884198 & 0.00198 & 2.239 & 13.04 \\
\hline 10 & 0.9599796 & 0.3898439 & 0.010687627 & -2.6028 & 0.000102240 & 0.000230 & 2.250 & 13.64 \\
\hline 12 & 0.9691552 & 0.3896591 & 0.003562430 & -2.6039 & 0.000011591 & 0.0000261 & 2.252 & 13.94 \\
\hline 14 & 0.9722041 & 0.3895975 & 0.001185071 & -2.5997 & 0.000001292 & 0.00000292 & 2.260 & 14.05 \\
\hline 16 & 0.9732169 & 0.3895771 & 0.000394367 & -2.5966 & 0.000000143 & 0.000000323 & 2.258 & 14.00 \\
\hline 18 & 0.9735537 & 0.3895703 & 0.000131304 & -2.5947 & 0.0000000160 & 0.0000000359 & 2.244 & 14.02 \\
\hline 20 & 0.9736657 & 0.3895679 & 0.000043726 & -2.5934 & 0.0000000020 & - & - & - \\
\hline 19 & 0.9738187 & 0.3895648 & 0.000075753 & -2.5934 & 0.0000000052 & - & - & - \\
\hline 17 & 0.9740123 & 0.3895604 & 0.000227232 & -2.5919 & 0.0000000471 & 0.0000001068 & 2.267 & 13.90 \\
\hline 15 & 0.9745936 & 0.3895491 & 0.000681373 & -2.5896 & 0.000000429 & 0.000000966 & 2.253 & 13.95 \\
\hline 13 & 0.9763339 & 0.3895136 & 0.002042434 & -2.5863 & 0.000003872 & 0.00000873 & 2.255 & 14.00 \\
\hline 11 & 0.9815535 & 0.3894076 & 0.006134795 & -2.5883 & 0.000035703 & 0.0000804 & 2.252 & 14.31 \\
\hline 9 & 0.9975391 & 0.3890762 & 0.018814380 & -2.6448 & 0.000376967 & 0.000848 & 2.249 & 16.76 \\
\hline 7 & -1.0471654 & 0.3870327 & 0.073047582 & -3.4214 & 0.027566087 & 0.045082622 & 1.635 & 98.90 \\
\hline
\end{tabular}



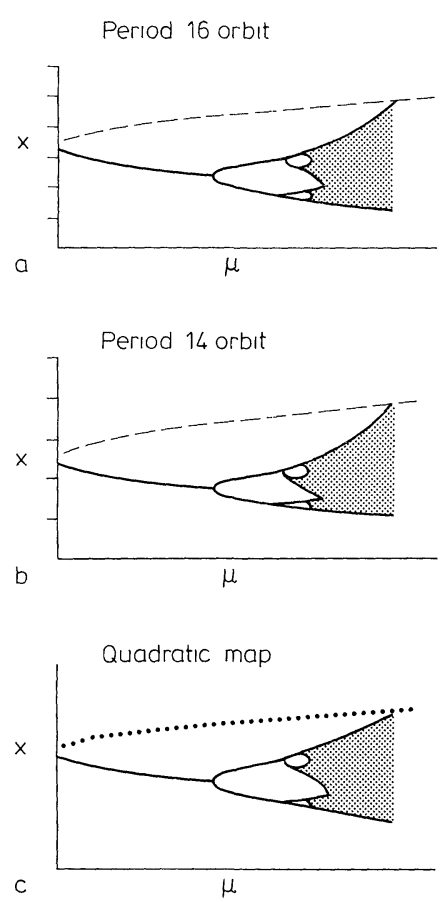

Fig. 7.1A-C. Bifurcation diagrams of: $A$ 16-piece simple attractor for the Hénon model at $B=0.3, x \in[-0.97323,-0.97320], \mu \in[1.15317543,1.15317579]$. B 14-piece simple attractor for the Hénon model at $B=0.3, x \in[-0.97225,-0.97215], \mu \in[1.1423847,1.1423879]$. C Quadratic map of the interval $x_{n+1}=\mu-x_{n}^{2}, x \in[3.5,-3.5], \mu \in[-0.25,2.25]$

Jacobian of (7.1) is negative. For our orientation-reversing situation, the only simple orbits that can appear before the first tangency are of even period; this accounts for the interesting order of appearance shown in Table 1. We did not investigate whether additional simple orbits of even period occur after the tangency. The family of sinks that we study agrees with our asymptotic (as $n \rightarrow \infty)$ quantitative estimates already for relatively small periods as shown in Table 1. Moreover, the qualitative behavior of the quadratic map of the interval seems to govern the evolution of simple attractors beyond what one would expect from the results in Sect. 4 (see Yorke et al., 1985). In Fig. 7.1 we compare the bifurcation diagrams of two simple attractors for the Hénon model, and of the quadratic map, in a striking qualitative and quantitative resemblance. Analogous pictures are exhibited by all the attractors listed in Table 1; in the same table we list various critical values for the attractors. The study was performed on an HP 85, a desk-top computer. We determined the duration of the $n$-piece attractor within three significant digits : more precision in the critical value $\mu_{c}$ is beyond feasibility for this particular device.

Acknowledgements. We would like to thank L. Nusse and S. Pelikan for their comments and suggestions. 


\section{References}

Belitskii, G.R.: Equivalence and normal forms of germs of smooth mappings. Russ. Math. Surveys 33, 107-177 (1978)

Bowen, R.: A Horseshoe with positive measures. Invent. Math. 29, 203-204 (1975)

Bowen, R., Ruelle, D.: The ergodic theory of axiom a flows. Invent. Math. 9, 181-202 (1975)

Chow, S.N., Hale, J.K.: Methods of bifurcation theory. Berlin, Heidelberg, New York: Springer 1982

Franceschini, V., Russo, L.: Stable and unstable manifolds of the Hénon mapping. J. Stat. Phys. 25, 757-769 (1981)

Gambaudo, J.M., Tresser, C.: Simple models for bifurcations creating horseshoes. J. Stat. Phys. 32, 455-476 (1983)

Gavrilov, N.K., Shilnikov, L.P.: On three-dimensional dynamical systems close to systems with a structurally unstable homoclinic curve I. Math. USSR Sb. 17, 467-485 (1972) and Part II, USSR Sb. 19, 139-156 (1973)

Guckenheimer, J., Holmes, P.: Nonlinear oscillations, dynamical systems, and bifurcation of vector field. Berlin, Heidelberg, New York: Springer 1983

Henry, B.: Escape from the unit interval under the transformation $x \rightarrow \lambda x(1-x)$. Proc. Am. Math. Soc. 41, 146-150 (1973)

Misiurewicz, M., Szewc, B.: Existence of a homoclinic point for the Hénon map. Commun. Math. Phys. 75, 285-291 (1980)

Nitecki, Z.: Differentiable dynamics. Cambridge, MA: MIT Press 1971

Newhouse, S.E.: Diffeomorphisms with infinitely many sinks. Topology 13, 9-18 (1974)

Newhouse, S.E.: On the abundance of wild hyperbolic sets. Publ. Math. Inst. Hautes Etud. Sci. 50, 101-151 (1979)

Nüsse, E.: Chaos, yet no chance to get lost. Doctoral dissertation Rijksuniversiteit Te Utrecht (1983)

Robinson, C.: Bifurcations to infinitely many sinks. Commun. Math. Phys. 90, 433-459 (1983)

Van Striien, S.: On the bifurcations creating horseshoes. Lecture Notes in Mathematics, Vol. 898, pp. 316-351. Rand, D., Young, L.S. (eds.), Berlin, Heidelberg, New York: Springer 1980

Yorke, J.A., Grebogi, C., Ott, E., Tedeschini-Lalli, L.: Scaling behavior of windows in dissipative dynamical systems. Phys. Rev. Lett. 54, 1095-1098 (1985)

Yorke, J.A., Alligood, K.T.: Periodic-doubling bifurcation: a prerequisite for horseshoes. Bull. Am. Math. Soc. 9, 319-322 (1983) and Commun. Math. Phys. 101, 305-321 (1985)

Communicated by Ya. G. Sinai

Received January 20, 1986 
